\title{
A piezoelectric tracked vehicle with potential application to planetary exploration
}

\author{
JIN JiaMei*, QIAN Fu, YANG Ying, ZHANG JianHui \& ZHU KongJun \\ State Key Laboratory of Mechanics and Control of Mechanical Structures, Nanjing University of Aeronautics and Astronautics, Nanjing 210016, \\ China
}

Received October 9, 2011; accepted November 17, 2011

\begin{abstract}
A piezoelectric driving method for rover vehicles is proposed in this paper. Employing this method, a tracked vehicle driven by friction forces from a frame mounted with piezoelectric elements was developed. The vehicle is designed with no driver sprocket, no idler-wheel and no supporting bogie wheels, and the vehicle thus requires no lubrication and has potential application in planetary exploration. The frame consists of a pair of piezoelectric transducers. Each transducer comprises four annular parts jointed by beams adhered with piezoelectric ceramics. The tracks are set to the outer surface of the annular parts by means of track tension. Traveling rotating waves are generated by piezoelectric transducers in the annular parts, which generate microscopic elliptical motions at the interface of the tracks. The microscopic elliptical motions from the piezoelectric transducers drive the track vehicle to move. Finite elements analysis was carried out to verify the operation principle using commercial software ANSYS. Piezoelectric transducers were fabricated, assembled and tested to validate the concepts of the proposed rover vehicle and confirm the simulation results. A prototype vehicle with mass of $0.57 \mathrm{~kg}$ moves at a speed of $4.3 \mathrm{~mm} / \mathrm{s}$ at a driving voltage of $250 \mathrm{~V}$ and operating frequency of $65.53 \mathrm{kHz}$.
\end{abstract}

rover vehicle, tracked vehicle, piezoelectric driving, mode, friction force

Citation: Jin J M, Qian F, Yang Y, et al. A piezoelectric tracked vehicle with potential application to planetary exploration. Chin Sci Bull, 2012, 57: 1339-1342, doi: 10.1007/s11434-012-5004-7

Robotic exploration of the lunar surface will provide important scientific data on climate, life history, and geologic resources. Landers and rovers for lunar exploration have been researched in recent decades. The main aim of research into lunar robotics exploration is to demonstrate technologies suitable for exploration of the lunar surface, including driving technologies for rover vehicles. Efficient rover vehicles that are compact, lightweight and consume little power are needed to meet the needs of lunar missions [1]. Five classes of locomotion systems are applicable to lunar/planetary exploration rovers: wheel, track, leg, bodyarticulation, and non-contact locomotion systems [2]. The chief advantage of legged robots is that they only need to overcome compaction resistance at the point of contact while wheeled and tracked robots that are considerably

*Corresponding author (email: jjm345@ sohu.com) more complex than algorithms for wheeled and tracked systems. Among the above mentioned types of mobility system, wheeled and tracked systems have been considered the main candidates for planetary exploration, especially the exploration of Mars, in recent decades wide applications on Earth.

The tracked (or caterpillar) concept is promising in that it offers low maximum ground pressure to promote vehicle performance (especially in the case of military vehicles) [3]. Tracks are generally preferred for general off-road applications as they offer low vehicle sinkage and thus low resistance to motion. Tracked vehicles have similar properties to wheeled vehicles and are generally favored for their higher tractional effort over more rugged terrain as they spread the load over a much wider area; a tracked vehicle will almost invariably offer greater drawbar pull than a four-, six-, or eight-wheeled vehicle of the same mass. A 
traction system has been adopted on the European Space Agency's Nanokhod micro-rover. The Nanokhod tracks were enclosed around the locomotion units to seal against dust, were able to climb obstacles rising $0.1 \mathrm{~m}$, had a speed of $5 \mathrm{~m} / \mathrm{h}$, and received power and data via a cable [4]. Clearly, tracks increase the ground contact area, which is maximized if the tracks follow the contours of the terrain, and tracked systems are superior in performance to wheeled vehicles by virtue of their distribution of weight over a large ground contact area. Tracked vehicles generally comprise a tread, a drive sprocket, an idler-wheel (or alternatively a powered wheel) and optionally a number of supporting bogie wheels. However, the design is too complex to apply on a planet. Many issues must be considered in detail, including the high power consumption due to inefficiency arising from friction between the track and the ground and friction between the large number of bogie wheels that maintain the track profile against the ground.

An emerging actuation technology of ultrasonic motors, which are compact and light motors having many advantages, is a candidate technology for rover vehicles $[5,6]$. The motors have high torque density at low speed, high holding torque, simple construction, and quick response, and can be made in many shapes, including annular, bar, disk and irregular shapes [7-9]. Currently, issues associated with the interface, longevity, efficiency and miniaturization of such motors are being studied. To ensure materials are suitable for harsh space environments and demanding space applications, rigorous analytical tools and thorough investigation are needed to determine the effects of extreme temperatures and vacuum conditions [10]. Furthermore, an important issue is that the mechanics requires lubricants, and the volatilization and deflation of lubricants in a vacuum are inevitable; thus, to the mechanics are hindered by the friction and wear (tear) of sliders and bearings. To solve this problem, effective driving and transport mechanics with compact construction and a low tear rate are being sought.

A novel tracked vehicle that is simple and compact and driven by friction forces from a frame with piezoelectric elements is designed in support of this objective. The vehicle is driven by a pair of piezoelectric vibrators, each of which is composed of four annular parts jointed by beams adhered with piezoelectric elements. The tracks are set along the outlet surfaces of the annular parts by means of track tension. Traveling rotating waves are generated by piezoelectric transducers in the annular parts along the beams, and they create microscopic elliptical motions at the interface of the tracks. The microscopic elliptical motions from the piezoelectric transducers drive the tracked vehicle to move. There are (1) no sliders or bearings in the tracked vehicle, which eliminates many of the disadvantages associated with the high internal friction and mechanical complexity of traditional tracks, (2) no bogie wheels, which reduces the internal friction losses that are characteristic of most traditional tracked vehicles, (3) no track links, which eliminates the catastrophic link breakage problem, and (4) no lubricants, which eliminates the problem of lubricant volatilization and deflation.

\section{Structure and principle}

The proposed vehicle is a novel tracked vehicle that has the potential of high mobility. As shown in Figure 1, the vehicle has three main parts: an aluminum vehicle frame, a pair of piezoelectric transducers and a pair of tracks (or caterpillars) made from rubber. Each transducer comprises a parallelogram beam and four annular parts at its four vertices. Leadzirconium titanate-based piezoelectric plates with identical thickness and section area are bonded on the external surfaces of bronze beams and their poling direction is the thickness direction. A highly elastic stainless-steel band is bonded to the inner surface of the track connected with the four annular parts under pre-pressure applied by a tensioner. The steel band is thin enough to be considered to bend freely. This structure does not have a driver sprocket, idler-wheel and supporting bogie wheels. The design substantially reduces the power requirement in comparison with traditional tracks. Instead of six motors for a sixwheeled vehicle, only two transducers are required for the proposed vehicle, and the number of parts is expected to be much lower than the numbers of parts in alternative designs. More importantly, because of the absence of a sliding interface in the vehicle frame, the track no longer needs to be sealed to protect exposed parts. The tight fit between the transducer and the track is limited to the annular portion of the transducers, which reduces the possibility of jamming by soil and rock particles. The ultrasonic-frequency vibration of the annular parts during motion keeps small particles away from the vehicle. The transducer/track contact is maintained by the tensioner under variable loads aided by a spring. The chief advantage of the tracked vehicle is that the ground pressure is distributed over a large footprint. The continuous track solves problems of high internal friction and mechanical complexity completely by avoiding link breakage relating to bogie wheels. It also makes for the easy climbing of obstacles by simply pitching the nose up and driving the chassis forward.

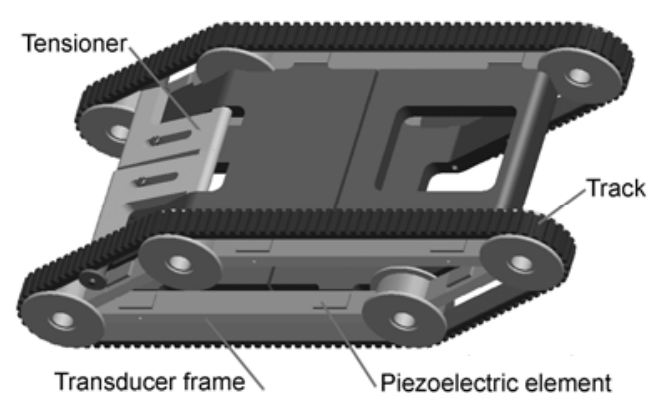

Figure 1 Tracked-vehicle structure. 
The driving principle of tracks through the use of a piezoelectric transducer is elucidated as follows. The working modes of the piezoelectric transducer are degenerate modes that exist in ring vibrators. The extensional mode has been used in the same situation as it has been used in ultrasonic motors [11]. The traveling wave along the circumference of the ring is obtained by driving two standing waves that are temporally and spatially phase shifted by $\pi / 2$ from each other. Bolted Langevin transducers, designed to excite radial nonaxisymmetric modes in the ring, have been used as a vibration generator [12]. A traveling-wave ultrasonic motor has also been developed with an annular part and a barshaped transducer. An experimental investigation has revealed that the traveling wave on the annular part can be excited by a bar-shaped transducer to drive the rotor motion [13]. The transducer described in this paper is shown in Figure 2, with each ring adhering to two beams. In our design, the angle included between adjacent beams is $\pi / 6$ or $5 \pi / 6$. The result is that two $\mathrm{B}_{03}$ bending modes in the annular parts with $\pi / 2$ spatial phase difference can be excited by the longitudinal vibration of two beams, as shown in Figure 3.

Traveling waves in annular parts are realized by synthesizing two standing waves excited by two sets of piezoelectric elements on the two pairs of beams. One set excites the longitudinal vibration in the horizontal beams and the other excites the longitudinal vibration in the oblique beams. When the set of piezoelectric plates on the horizontal beams are supplied with sine voltage, the beams exhibit third-order longitudinal vibration $\left(\mathrm{L}_{3}\right)$. Consequently, the annular parts

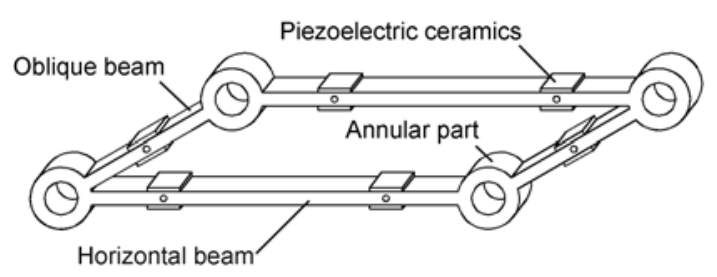

Figure 2 Transducer structure.
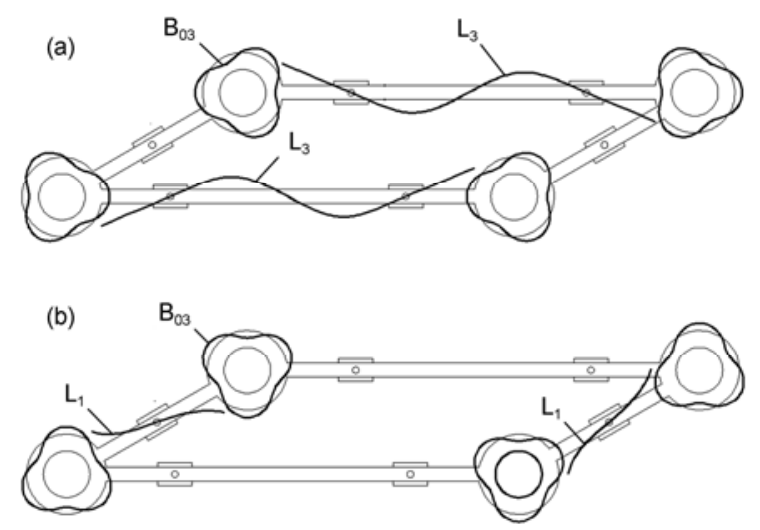

Figure 3 Operating $\mathrm{B}_{03}$ modes with (a) third-order longitudinal vibration of the horizontal beams and (b) with first-order longitudinal vibration of the oblique beams. vibrate in $\mathrm{B}_{03}$ mode, as shown in Figure 3(a). When the set of piezoelectric plates on the oblique beams are supplied with cosine voltage, the beams exhibit first-order longitudinal vibration $\left(\mathrm{L}_{1}\right)$, which leads to the annular parts vibrating with $\mathrm{B}_{03}$ mode in a different phase, as shown in Figure 3(b).

Finite element analysis was carried out using the commercial code ANSYS to simulate the traveling wave in the annular parts. The modal analysis illustrates that $\mathrm{B}_{03}$ mode vibration appears in the annular parts when the horizontal beams vibrate in the third-order longitudinal mode at their eigenfrequency $\omega$, as shown in Figure 4(a). Additionally, $\mathrm{B}_{03}$ mode vibration appears in the annular parts when the oblique beams vibrate in the first-order longitudinal mode near their eigenfrequency $\omega$, but with a spatial phase difference of $\pi / 2$ relative to the former vibration, as shown in Figure 4(b). The harmonic analysis verifies that rotary traveling waves are generated in the annular parts and each particle in the surface follows an elliptical trajectory as the two sets of piezoelectric ceramics are separately supplied with sine and cosine voltages.

\section{Prototype and experiments}

Piezoelectric transducers were built according to the finite element simulation results. The transducer has an annular outer diameter of $19.6 \mathrm{~mm}$ and an inner diameter of 11.8 $\mathrm{mm}$. The central distance of adjacent annular parts is 50 or $102 \mathrm{~mm}$, respectively. The beam has a section of $8 \mathrm{~mm} \times$ $3.6 \mathrm{~mm}$. Each piezoelectric ceramic (lead zirconium titanate) plate coated with a silver electrode has dimensions of $10 \mathrm{~mm}$ $\times 5 \mathrm{~mm} \times 0.5 \mathrm{~mm}$ and electric parameters of $d_{33}=280, K_{\mathrm{p}}=$ 0.31 , and $Q_{\mathrm{m}}=1500$. The vibration modes of the assembled transducers were measured with an OFV056 Doppler laser vibrometer (Polytec). A distinct $\mathrm{B}_{30}$ bending mode with resonant frequency of $65.48 \mathrm{kHz}$ was found and the resonant frequency of $\mathrm{B}_{30}$ bending mode was $65.58 \mathrm{kHz}$, as shown in Figure 5.

A prototype rover vehicle, with mass of $0.57 \mathrm{~kg}$, was

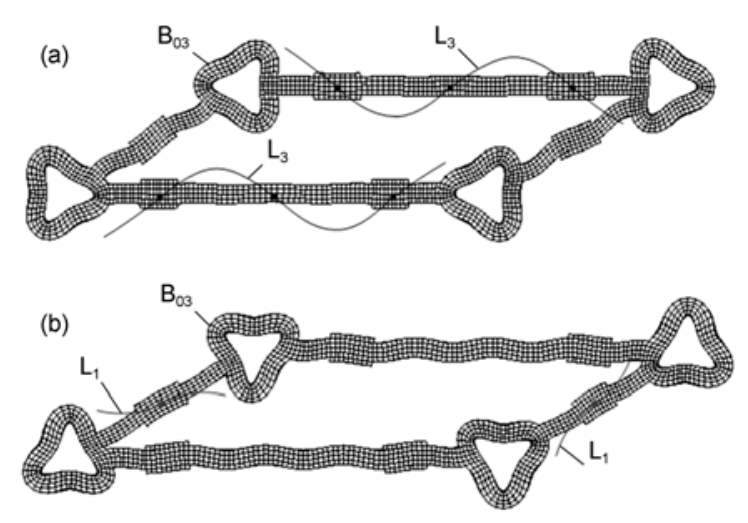

Figure 4 Finite element modal analysis with (a) third-order longitudinal vibration of the horizontal beams and (b) first-order longitudinal vibration of the oblique beams. 

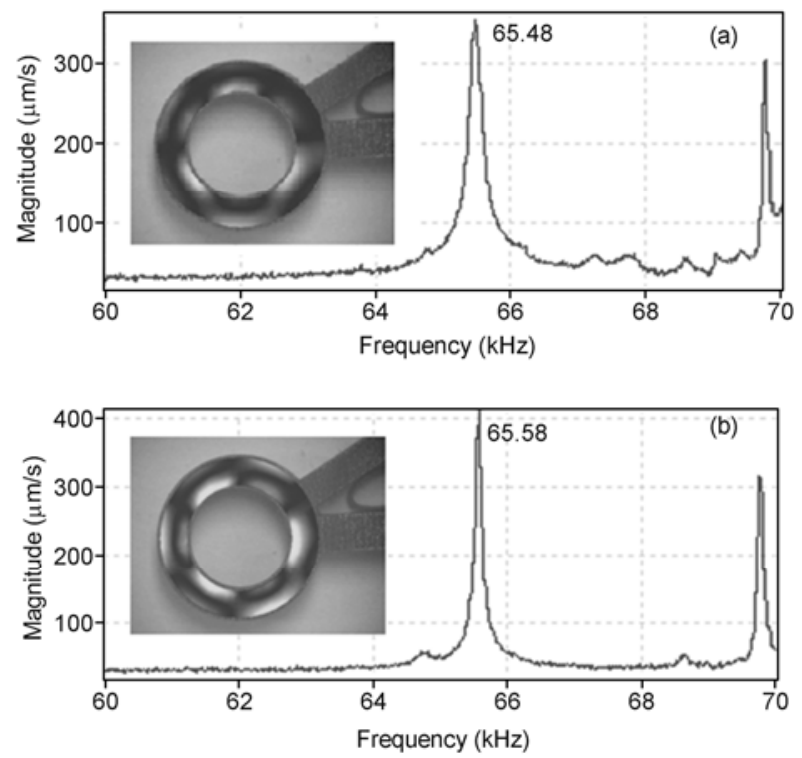

Figure 5 Modal test using an OFV056 Doppler laser vibrometer (Polytec) excited by (a) third-order longitudinal vibration in the horizontal beams and (b) first-order longitudinal vibration in the oblique beams.

built. Applying a pair of sinusoidal drive voltages of $250 \mathrm{~V}$ with $\pi / 2$ phase shift and frequency of $65.53 \mathrm{kHz}$ to the piezoelectric ceramics, the moving speed of the vehicle reached $4.3 \mathrm{~mm} / \mathrm{s}$.

\section{Summary}

This paper proposed a novel piezoelectric method for driving tracked vehicles. A tracked vehicle driven by piezoelectric ceramics developed for planetary exploration has the characteristics of (1) a simple and compact structure with no driver sprocket, no idler-wheel and no supporting bogie wheels, (2) little internal friction loss, since there is no sliding interface, and no seal requirement to protect the exposed parts from dust, (3) no lubrication, which eliminates the problem of lubrication volatilization and deflation, and (4) efficient energy use, being able to self-hold when the power is off. A prototype of the tracked vehicle achieved a traveling speed of $4.3 \mathrm{~mm} / \mathrm{s}$ and had a mass of $0.57 \mathrm{~kg}$. It was confirmed that the piezoelectric transducer can be applied effectively to the motion mechanism of a vehicle. Additionally, the tracked vehicle may have applications to locomo- bile systems that require a compact structure and low cost.

This study was supported by the National Natural Science Foundation of China (5117702, 91023020), NFSC Guangdong Joint Fund (U0934004), and Program for New Century Excellent Talents in University (NCET-10- 0070).

1 Das H, Bao X, Bar-Cohen Y, et al. Robot manipulator technologies for planetary exploration. In: Wereley N M, ed. Proceedings of the 6th Annual International Symposium on Smart Structures and Materials, 1999 Mar 01-04, Newport Beach. Bellingham: Society of Photo-Optical Instrumentation Engineers, 1999. 175-182

2 Ellery A. Environment - robot interaction - the basis for mobility in planetary micro-rovers. Rob Autom Syst, 2005, 51: 29-39

3 Hetherington J. Applicability of the MMP concept in specifying off-road mobility for wheeled and tracked vehicles. J Terramech, 2001, 38: 63-70

4 Bertrand R, Bruckner J, van Winnendael M. The Nanokhod microrover-A versatile platform for surface exploration of celestial bodies. In: Foing B H, Perry M, eds. 4th International Conference on Exploration and Utilisation of the Moon (ICEUM 4), 2000 Jul 10-14, Noordwijk. Noordwijk: ESA PUBLICATIONS DIVISION C/O ESTEC, 2000. 149-154

5 Kubota T, Tada K, Kunii Y. Smart manipulator actuated by ultrasonic motors for lunar exploration. In: Proceedings of IEEE International Conference on Robotics and Automation: The Half-Day Workshop on: Towards Autonomous Agriculture of Tomorrow, 2008 May 19-23, Pasadena. Piscataway: Institute of Electrical and Electronics Engineers Inc, 2008. 3576-3581

6 Kunii Y, Tada K, Kuroda Y, et al. Micromanipulator with lightweight and low power consumption by using ultra-sonic motor for planetary explore mission. In: Proceedings of 10th IEEE International Conference on Advanced Robotics (ICAR 2001), the fundamentals: From present to tomorrow, 2001 Aug 22-25, Budapest. Budapest: Budapest Polytechnic, 2001. 457-462

7 Ueha S, Tomikawa Y. Ultrasonic Motors Theory and Applications. Oxford: Clarendon Press, 1993

8 Zhou T Y, Zhang Y, Chen Y, et al. A nut-type ultrasonic motor and its application in the focus system. Chin Sci Bull, 2009, 54: 37783783

9 Zhou T Y, Zhang K, Chen Y, et al. A cylindrical rod ultrasonic motor with $1 \mathrm{~mm}$ diameter and its application in endoscopic OCT. Chin Sci Bull, 2005, 50: 826-830

10 Zhao C S. Ultrasonic Motors Technologies and Applications. Beijing: Springer and Science Press, 2010

11 Iwamatsu S, Ueha S, Kuribayashi M, et al. Rotary ultrasonic motor using extensional vibration of a ring. Jpn J Appl Phys Suppl, 1986, 25: $174-176$

12 Iula A, Corbo A, Pappalardo M. Influence of the number of driving transducers on the performance of a high power traveling wave ultrasonic motor. In: IEEE, ed. Proceedings of IEEE Ultrasonics Symposium, 2006 Oct 03-06, Vancouver. Piscataway: Institute of Electrical and Electronics Engineers Inc., 2006. 160-163

13 Jin J M, Zhao C S. A novel traveling wave ultrasonic motor using a bar shaped transducer. J Wuhan Univ Technol Mater Sci Ed, 2008, 23: 961-963

Open Access This article is distributed under the terms of the Creative Commons Attribution License which permits any use, distribution, and reproduction in any medium, provided the original author(s) and source are credited. 